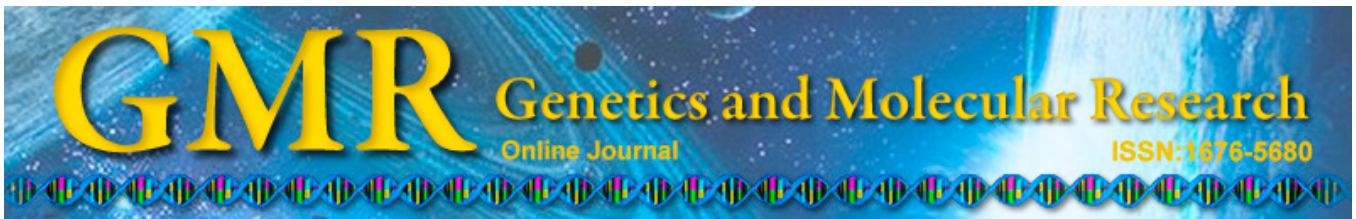

\title{
CYFRA21-1 levels could be a biomarker for bladder cancer: a meta-analysis
}

\author{
L.I. Kuang1, W.J. Song ${ }^{2}$, H.M. Qing ${ }^{1}$, S. Yan $^{2}$ and F.L. Song ${ }^{1}$ \\ ${ }^{1}$ Department of Pathology, \\ General Hospital of Shenyang Military Area Command of Chinese PLA, \\ Shenyang, Liaoning, China \\ ${ }^{2}$ Department of Urological Surgery, Red Cross Hospital of Shenyang, \\ Liaoning, China \\ Corresponding author: F.L. Song \\ E-mail:mac_k1@126.com
}

Genet. Mol. Res. 14 (2): 3921-3931 (2015)

Received April 11, 2014

Accepted October 20, 2014

Published April 27, 2015

DOI http://dx.doi.org/10.4238/2015.April.27.6

\begin{abstract}
The proteolytic region of cytokeratin-19, referred to as CYFRA21-1, is a soluble molecule present in the serum and other body fluids, and is considered a tumor marker in several neoplastic diseases. To examine whether urinary or serum samples containing CYFRA21-1 can be used as biomarkers for bladder cancer, we conducted a comprehensive meta-analysis of 3 case-control studies. In all studies considered, patients with bladder cancer had a higher CYFRA21-1 level than healthy subjects. Subgroup analysis showed that patients with metastatic bladder cancer had a higher CYFRA21-1 level than those with locally invasive disease. However, no significant difference in CYFRA21-1 was observed between patients with stage I and stage II bladder cancer; there was also no difference in patients with stage II local bladder cancer and those with stage III local bladder cancer. Based on our results, CYFRA21-1 level may be a diagnostic biomarker for diagnosing bladder cancer as well as a possible biomarker for differentiation between local and metastatic bladder cancer. However, it cannot be used as a urinary or serum biomarker for differentiating
\end{abstract}


histological stages of local bladder cancer for histological grades I-III.

Key words: Bladder cancer; CYFRA21-1; Meta-analysis

\section{INTRODUCTION}

Bladder cancer is among the 10 most frequent cancers worldwide. Diagnostic procedures in patients with bladder cancer symptoms include urine cytology, cystoscopy with biopsy, and excretory urography, but cystoscopy remains the reference method for detecting primary or recurrent transitional cell carcinoma (Raghavan et al., 1990). Depth of muscle invasion is used to classify the clinical stage, and differentiation and histological characteristics are used for tumor grading. Treatment and prognosis depend on these latter features (Griffiths and Neal, 1996). Superficial tumors are transurethral resected and may also receive intravesical chemotherapy (Newling, 1996), while invasive tumors are deep-resected and treated with systemic therapy, and cystestomized (Javle and Raghavan, 1996) and metastatic tumors are treated with systemic chemotherapy. More than $50 \%$ of superficial tumors recur within 5 years, and $10-20 \%$ of these progress into invasive disease. Prognosis is multifactorial, and regular follow-up is required (Morris et al., 1995). Cystoscopy is an invasive and uncomfortable procedure; therefore, many investigators have attempted to develop alternative methods (Nisman et al., 2009). Cytology is a diagnostic modality that can replace cystoscopy, but its sensitivity is too low to detect low-grade neoplasms (Badalament et al., 1987). Considerable effort has been made to identify noninvasive biomarkers for bladder cancer with sufficient diagnostic ability.

Biomarkers can be specific cells, enzymes, hormones, genes, or gene products that can be detected and measured in parts of the body such as the blood, urine, or tissue (Bhide et al., 2013). Numerous reports have recently been published regarding serum tumor markers for bladder cancer. The proteolytic region of cytokeratin (CK)-19, referred to as CYFRA21-1, is a soluble molecule in the serum and other body fluids and has been used as a tumor marker in several neoplastic diseases. High levels of CYFRA21-1 have been observed in patients with solid tumors, including lung (Stieber et al., 1994), head and neck (Doweck et al., 1995), gastric (Nakata et al., 1996), ovarian (Hasholzner et al., 1994), breast (Nakata et al., 2000), and prostate cancers (Theyer et al., 1999). We hypothesized that measuring the urinary or serum levels of CYFRA21-1 would serve as a valuable biomarker for the diagnosis of bladder cancer. Furthermore, if CYFRA21-1 levels significantly differ in different clinical stage of bladder cancer, measuring CYFRA21-1 levels will be useful for diagnosis, assessment, follow-up, and recognition of bladder cancer recurrence. Therefore, we performed a meta-analysis that included the most recent and relevant articles to identify statistical evidence of the usefulness of CYFRA21-1 in bladder cancer.

\section{MATERIAL AND METHODS}

\section{Literature search}

We performed an electronic search of the PubMed, Cochrane library, Embase, Web of Science, Springer Link, and CBM databases extensively to identify relevant studies available through December 11, 2013. The search terms included ['bladder cancer' or 'bladder tumor' 
or 'bladder neoplasms' (Mesh)] and ['proteolytic part of CK-19' or 'CYFRA21-1' (Mesh)]. References in the eligible studies or textbooks were also reviewed through a manual search to identify other potentially eligible studies.

\section{Inclusion and exclusion criteria}

The studies included were required to meet the following criteria: i) the type of study should be a case-control study; ii) these case-control studies should focus on the association between CYFRA21-1 level and bladder cancer; iii) all patients were diagnosed with bladder cancer (diagnosis was based on cystoscopy, transurethral resection or cystectomy when applicable, and histopathological confirmation); iv) patient bladder tumors were graded (I, II, or III) according to the 1973 World Health Organization system. The publication language was restricted to English. Studies were excluded if they reported incomplete, useless, or overlapping data; if they used median and interquartile ranges to describe the CYFRA21-1 levels; or if they were meta-analyses, letters, reviews, or editorial articles.

\section{Data extraction}

Using a standardized form, data from published studies were extracted independently by 2 reviewers (Li Kuang and Wenjun Song) to populate the necessary information. The following information was extracted from each of the articles: first author, year of publication, study design, source of cases and controls, number of cases and controls, sample, clinical symptom, treatment, and CYFRA21-1 level. In case of conflicting evaluations, an agreement was reached following discussion with a third reviewer (Fulin Song).

\section{Quality assessment of studies included}

Two reviewers (Li Kuang and Wenjun Song) independently assessed the quality of papers according to modified STROBE quality score systems (von Elm et al., 2007; Zhang et al., 2011). Forty assessment items related to quality appraisal were used in this meta-analysis, with scores ranging from 0-40. Scores of 0-20, 20-30, and 30-40 were defined as low, moderate, and high quality, respectively. Disagreement was resolved by discussion.

\section{Statistical analysis}

The standardized mean difference (Std.MD) or mean difference (MD) and 95\% confidence interval $(95 \% \mathrm{CI})$ were calculated using Review Manager Version 5.1.6 (provided by the Cochrane Collaboration, available at: http://ims.cochrane.org/revman/download) and STATA Version 12.0 (StataCorp, College Station, TX, USA) software. Betweenstudy variations and heterogeneities were estimated using Cochran's Q-statistic (Higgins and Thompson, 2002; Zintzaras and Ioannidis, 2005) $(\mathrm{P} \leq 0.05$ was considered to indicate statistically significant heterogeneity). We also quantified the effect of heterogeneity using the $\mathrm{I}^{2}$ test, which ranged from $0-100 \%$ and represented the proportion of inter-study variability that can be attributed to heterogeneity rather than by chance. When a signifi- 
cant Q-test $(\mathrm{P} \leq 0.05)$ or $\mathrm{I}^{2}>50 \%$ indicated that heterogeneity among studies existed, the random effects model was conducted for meta-analysis. Otherwise, the fixed effects model was used. To establish the effect of heterogeneity on the conclusions of the metaanalyses, subgroup analysis was carried out. Funnel plots were used to detect publication bias. However, because of limitations caused by varied sample sizes and subjective reviews, Egger's linear regression test, which measures funnel plot asymmetry using a natural logarithm scale of the odds ratio (OR), was used to evaluate publication bias (Peters et al., 2006). When the $\mathrm{P}$ value was $<0.05$, publication bias was considered to be statistically significant. All P values were 2-sided. To ensure the reliability and accuracy of the results, 2 reviewers (Li Kuang and Wenjun Song) populated the data in the statistical software programs independently and obtained the same results.

\section{RESULTS}

\section{Characteristics of studies included}

According to the inclusion criteria, 3 studies (Sánchez-Carbayo et al., 1999; Andreadis et al., 2005; Washino et al., 2011) met the inclusion criteria and were included in the metaanalysis. The flow chart of study selection is shown in Figure 1. The 3 case-control studies included 227 patients with bladder cancer ( 97 patients diagnosed with locally invasive bladder cancer and 99 with metastatic bladder cancer histological stages I, II, and III, including 39,60 , and 68 patients, respectively), as well as 63 controls. The publication years of the studies ranged from 1999 to 2010. All patients were diagnosed with bladder cancer based on cystoscopy, transurethral resection or cystectomy when applicable, and histopathological confirmation. Control subjects were healthy individuals. All quality scores of studies included were $>20$ (moderate-high quality). The characteristics and methodological quality of the studies included are summarized in Table 1.

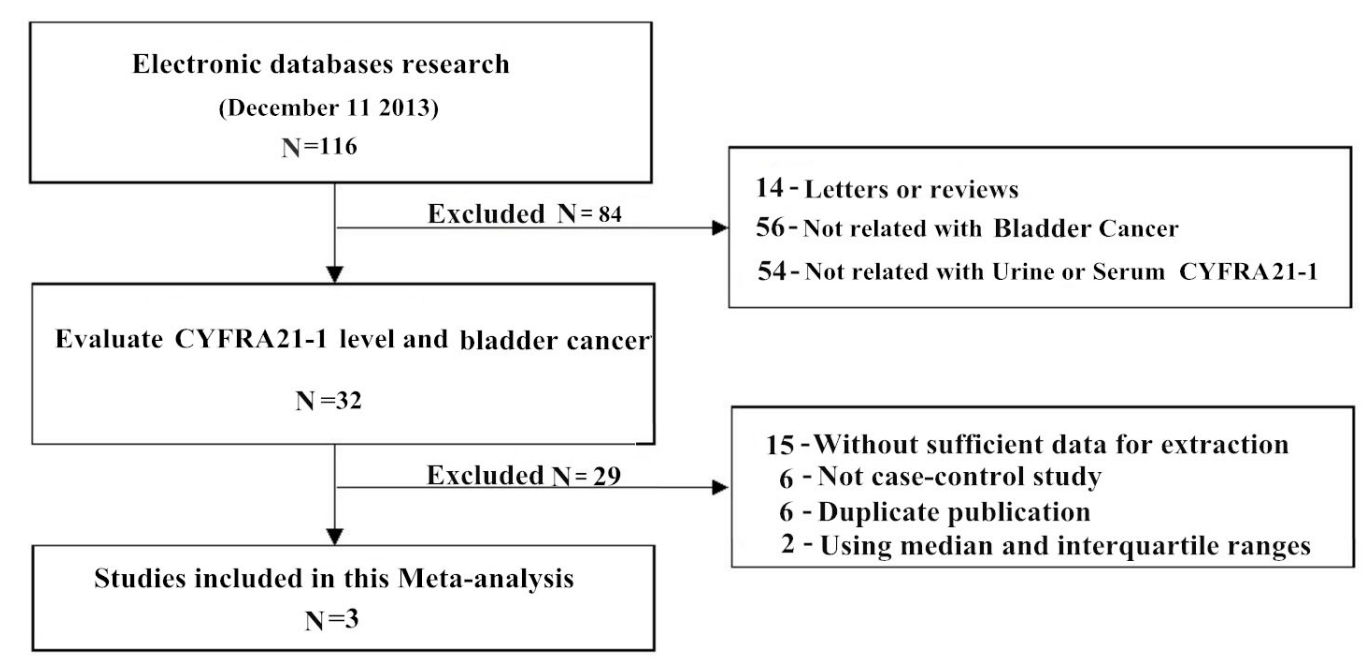

Figure 1. Flow chart showing study selection procedure. 
CYFRA21-1 levels and bladder cancer risk

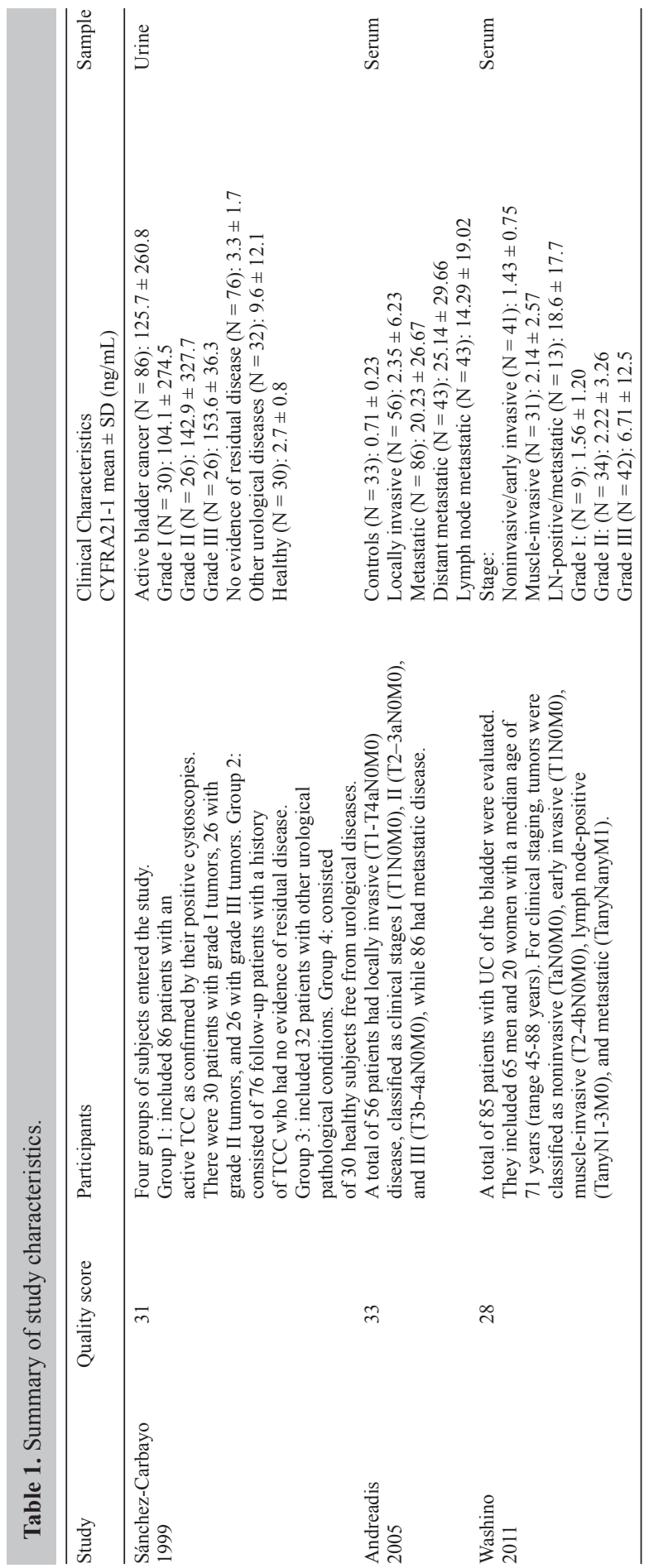




\section{Difference between patients with bladder cancer and healthy controls in CYFRA21-1 levels}

A summary of the meta-analysis findings of the difference in CYFRA21-1 levels between patients with bladder cancer and healthy controls is provided in Figure 2. The metaanalysis result showed that patients with bladder cancer had higher CYFRA21-1 levels than healthy controls (Std.MD $=0.70,95 \% \mathrm{CI}=0.40-1.00, \mathrm{P}<0.00001)$. Sensitivity analysis was conducted by omitting single studies, and no influence was observed in the significance of the pooled Std.MD.

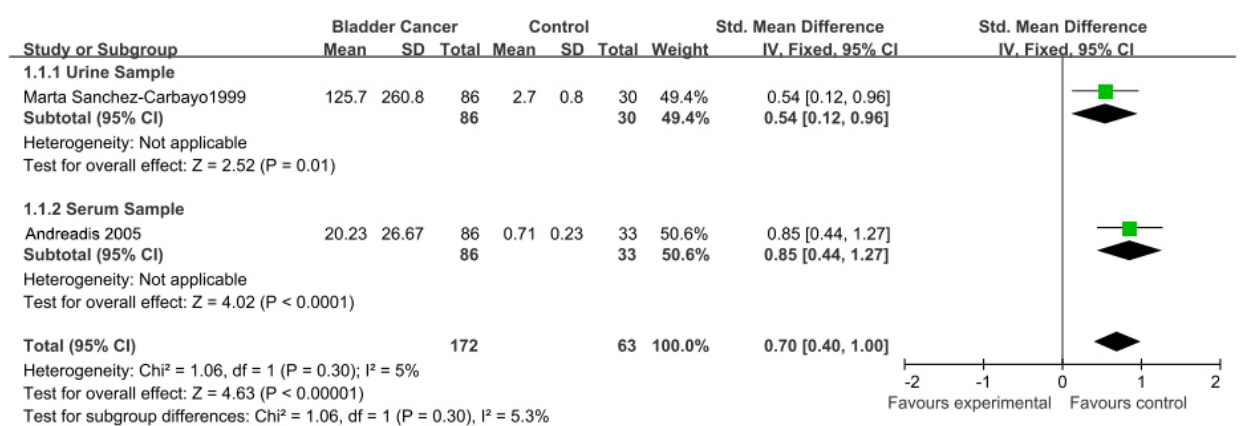

Figure 2. Association between CYFRA21-1 levels and bladder cancer risk. 95\% CI = 95\% confidence interval; $\mathrm{df}$ $=$ degrees of freedom.

\section{Difference between patients with bladder cancer histological stages I and II in CYFRA21-1 levels}

A summary of the meta-analysis findings regarding the difference in CYFRA21-1 levels between patients with bladder cancer histological stages I and II is shown in Figure 3. The meta-analysis results revealed no significant difference between patients with bladder cancer histological stages I and II for CYFRA21-1 levels $(\mathrm{P}=0.47)$. Sensitivity analysis was conducted by omitting single studies, and no influence was found in the significance of the pooled Std.MD.

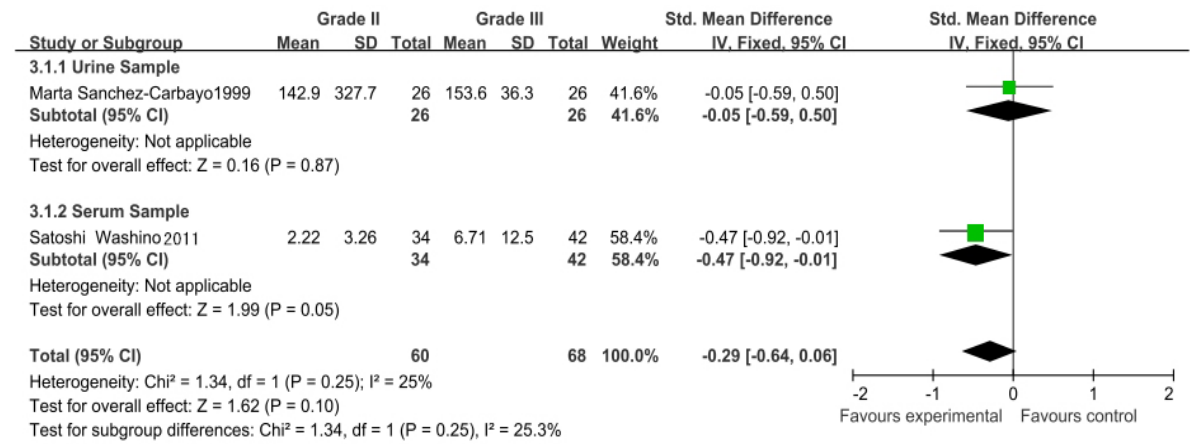

Figure 3. CYFRA21-1 levels with bladder cancer histological stages I and II. 95\% CI $=95 \%$ confidence interval; $\mathrm{df}=$ degrees of freedom. 


\section{Difference between patients with bladder cancer histological stages II and III in CYFRA21-1 levels}

A summary of the meta-analysis findings of the difference in CYFRA21-1 levels between patients with bladder cancer histological stages II and III is shown in Figure 4. The meta-analysis results showed that no significant difference was found between patients with bladder cancer histological stages II and III in CYFRA21-1 levels $(\mathrm{P}=0.10)$. Sensitivity analysis was conducted by omitting single studies, and no influence was observed in the significance of the pooled Std.MD.

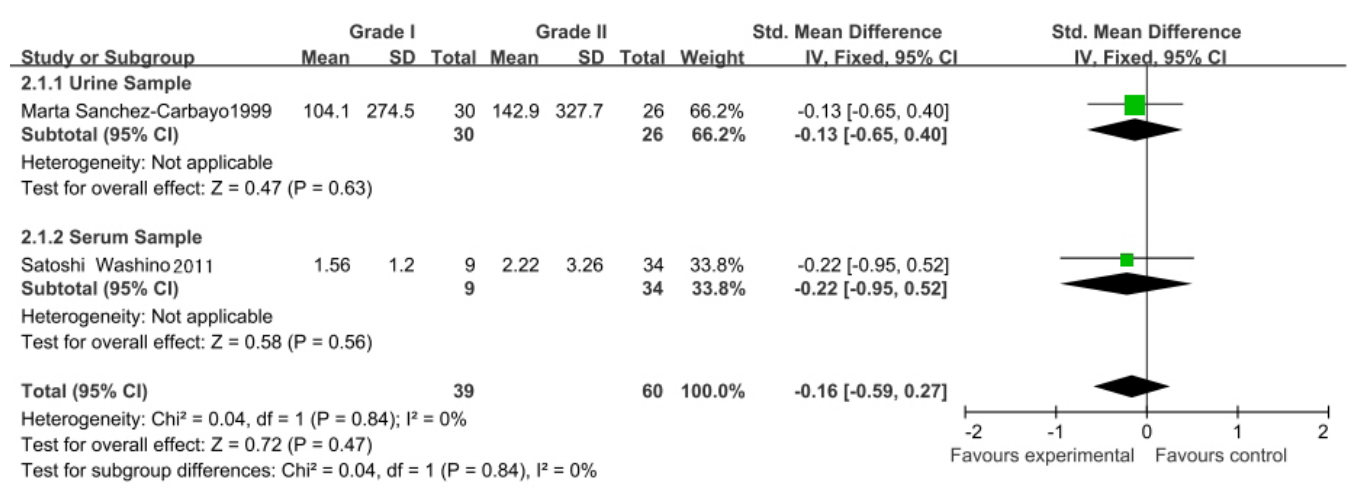

Figure 4. CYFRA21-1 levels with bladder cancer histological stages II and III. 95\%CI $=95 \%$ confidence interval; $\mathrm{df}=$ degrees of freedom.

\section{Difference in CYFRA21-1 levels between patients with locally invasive bladder cancer and metastatic bladder cancer}

A summary of the meta-analysis findings of the difference in CYFRA21-1 levels between patients with locally invasive bladder cancer and metastatic bladder cancer is shown in Figure 5. The meta-analysis result showed that patients with locally invasive bladder cancer had lower CYFRA21-1 levels than those with metastatic bladder cancer $(\mathrm{MD}=-17.69,95 \% \mathrm{CI}=-22.70-12.68, \mathrm{P}<0.00001)$. Sensitivity analysis was conducted by omitting single studies, and no influence was found in the significance of the pooled MD.

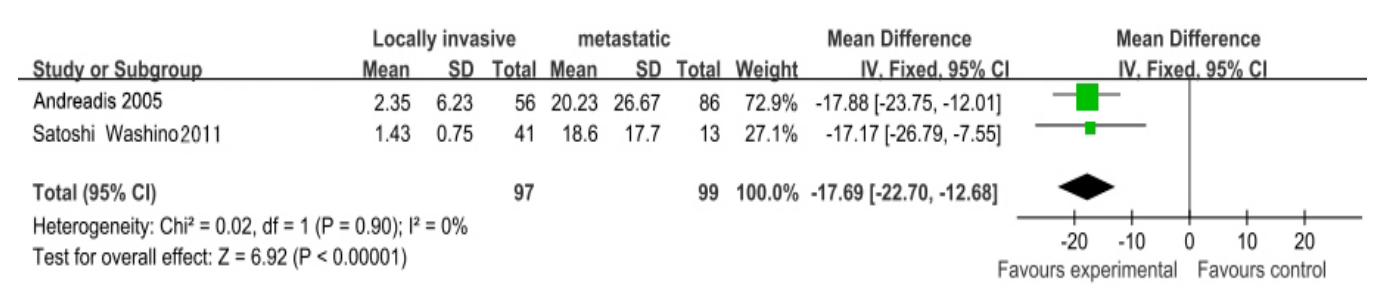

Figure 5. CYFRA21-1 levels with locally invasive bladder cancer and metastatic bladder cancer. 95\%CI $=95 \%$ confidence interval; $\mathrm{df}=$ degrees of freedom. 


\section{Publication bias}

Publication bias of the literature was assessed by Begger's funnel plot and the Egger linear regression test. The Egger linear regression test was used to measure funnel plot asymmetry. All graphical funnel plots of the studies included were symmetrical (Figures 6 and 7). The Egger test also showed that there was no statistical significance in all evaluations of publication bias (all $\mathrm{P}>0.05$ ).
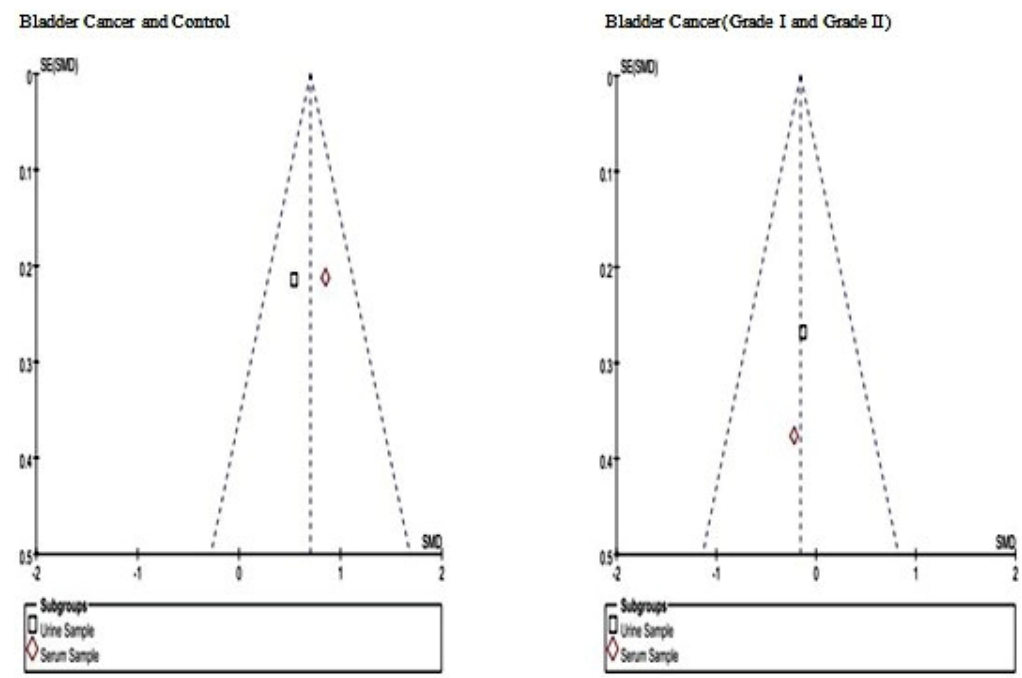

Figure 6. Begger funnel plot of publication bias based on CYFRA21-1 levels. SE $=$ standard error; SMD = standardized mean difference odds ratio.
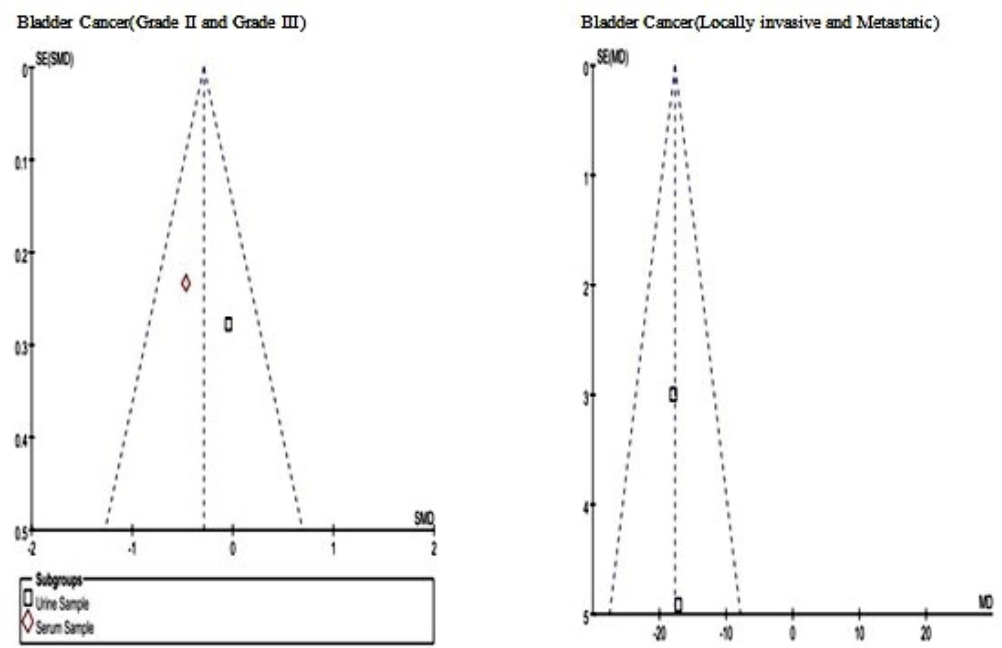

Figure 7. Begger funnel plot of publication bias based on CYFRA21-1 levels. SE $=$ standard error; SMD = standardized mean difference odds ratio. 


\section{DISCUSSION}

The high propensity for bladder cancer recurrence highlights the need for cystoscopic surveillance at defined intervals. Although flexible cystoscopy has greatly reduced the morbidity associated with cystoscopy, it remains an invasive and uncomfortable examination for the patient. Various non-invasive tests have been developed, including urine cytopathology, immunostaining, and fluorescence in situ hybridization of exfoliated cells or measuring the concentration of soluble components in the urine or serum such as CKs. In addition, bladder ultrasound scanning has been used to detect recurrent bladder tumors.

The development of bladder cancer includes an early step related to cellular damage, which may be followed by cellular differentiation and exophytic growth. During this process, bladder cells can be exfoliated into urine, and all of their intracellular components can be detected in urine-voided samples. CKs as intracellular filaments are thought to be present at different concentrations in the urine or serum of patients with bladder cancer, depending on their proliferation and production rates and the different degrees of exfoliation in the different subtypes of bladder cancer. CYFRA21-1 is a soluble fragment of CK-19, can be analyzed using an enzyme-linked immunosorbent assay, and is measurable in the serum and urine. In one study (Pariente et al., 2000), abnormal serum levels of CYFRA21-1 in patients with bladder cancer were observed only in patients with metastatic disease. In another study (Andreadis et al., 2005), patients with abnormal CYFRA21-1 levels showed a significantly worse overall median survival, which was correlated to the response to systemic treatment.

In our meta-analysis, we included 3 independent studies and examined the association between urinary or serum CYFRA21-1 level and bladder cancer. Overall, our analysis showed that patients with bladder cancer had a higher CYFRA21-1 levels than healthy subjects. CYFRA21-1 levels were higher in the bladder cancer group than in the total control group, which is consistent with the results of van Rhijn et al. (2005). This result suggested that CYFRA21-1 levels are a potential biomarker for the diagnosis of bladder cancer. In addition, we found that metastatic disease patients had significantly higher CYFRA21-1 levels than those with locally invasive disease. Therefore, CYFRA21-1 is a possible biomarker for differentiating between bladder cancer subtypes. However, we found no significant difference between CYFRA21-1 levels in patients with stage I local bladder cancer and stage II local bladder cancer. We also found no significant difference between CYFRA21-1 levels in patients with stage II local bladder cancer and stage III local bladder cancer. Thus, it is necessary to develop additional urinary biomarkers for the differential diagnosis of stage I local bladder cancer and stage II local bladder cancer as well as to differentiate between stage II local bladder cancer and stage III local bladder cancer.

There were several limitations to our study. First, the number of studies and subjects included in the analysis were small. Second, some relevant studies could not be included in our analysis because of incomplete raw data. Third, we could not address sources of heterogeneity among all studies. Fourth, although all cases and controls in each study were well-defined with similar inclusion criteria, there may be factors that were not taken into account, which may have influenced our results. Fifth, meta-analyses are retrospective studies that are subject to methodological limitations. Most importantly, our meta-analysis was based on unadjusted Std. MDs or MDs estimates because not all published studies presented adjusted Std.MDs or MDs or presented Std.MDs or MDs that were not adjusted by the same potential confounders, such as age, ethnicity, and urine collection timing. Thus, additional investigations in these areas are 
necessary, and our conclusions should be interpreted cautiously.

In conclusion, this meta-analysis of 3 case-control studies showed that CYFRA21-1 level may be a diagnostic biomarker for the diagnosis of bladder cancer as well as a possible biomarker for differentiating between bladder cancer subtypes (locally invasive or metastatic). However, it cannot be used as a biomarker to distinguish between stage I, II, and III bladder cancer. Because few studies have been conducted in this field, current evidence remains limited. Therefore, larger studies including proper control of confounding factors are necessary to obtain valid results.

\section{REFERENCES}

Andreadis C, Touloupidis S, Galaktidou G, Kortsaris AH, et al. (2005). Serum CYFRA21-1 in patients with invasive bladder cancer and its relevance as a tumor marker during chemotherapy. J. Urol. 174: 1771-1775.

Badalament RA, Hermansen DK, Kimmel M, Gay H, et al. (1987). The sensitivity of bladder wash flow cytometry, bladder wash cytology, and voided cytology in the detection of bladder carcinoma. Cancer 60: 1423-1427.

Bhide AA, Cartwright R, Khullar V and Digesu GA (2013). Biomarkers in overactive bladder. Int. Urogynecol. J. 24: 1065-1072.

Doweck, I, Barak M, Greenberg E, Uri N, et al. (1995). CYFRA21-1. A new potential tumor marker for squamous cell carcinoma of head and neck. Arch. Otolaryngol. Head Neck Surg. 121: 177-181.

Griffiths L and Neal DE (1996). Prognostic markers in bladder cancer. Curr. Opin. Urol. 6: 267-271.

Hasholzner U, Baumgartner L, Stieber P, Meier W, et al. (1994). Significance of the tumor markers CA 125 II, CA 72-4, CASA and CYFRA21-1 in ovarian carcinoma. Anticancer Res. 14: 2743-2746.

Higgins JP and Thompson SG (2002). Quantifying heterogeneity in a meta-analysis. Stat. Med. 21: 1539-1558.

Javle M and Raghavan D (1996). Progress in the treatment of invasive bladder cancer. Curr. Opin. Urol. 6: 280-285.

Morris SB, Gordon EM, Shearer RJ and Woodhouse CR (1995). Superficial bladder cancer: for how long should a tumorfree patient have check cystoscopies? Br. J. Urol. 75: 193-196.

Nakata B, Chung YS, Kato Y, Ogawa M, et al. (1996). Clinical significance of serum CYFRA21-1 in gastric cancer. $B r$. J. Cancer 73: 1529-1532.

Nakata B, Ogawa Y, Ishikawa T, Ikeda K, et al. (2000). Serum CYFRA21-1 is one of the most reliable tumor markers for breast carcinoma. Cancer 89: 1285-1290.

Newling DW (1996). Preventing recurrence and progression in superficial bladder cancer. Curr. Opin. Urol. 6: 272-275.

Nisman B, Yutkin V, Peretz T, Shaprio A, et al. (2009). The follow-up of patients with non-muscle-invasive bladder cancer by urine cytology, abdominal ultrasound and urine CYFRA21-1: a pilot study. Anticancer Res. 29: 4281-4285.

Pariente J, Bordenave L, Jacob F, Gobinet A, et al. (2000). Analytical and prospective evaluation of urinary cytokeratin 19 fragment in bladder cancer. J. Urol. 163: 1116-1119.

Peters JL, Sutton AJ, Jones DR, Abrams KR, et al. (2006). Comparison of two methods to detect publication bias in metaanalysis. JAMA 295: 676-680.

Raghavan D, Shipley WU, Garnick MB, Russell PJ, et al. (1990). Biology and management of bladder cancer. New Engl. J. Med. 322: 1129-1138.

Sánchez-Carbayo M, Espasa A, Chinchilla V, Herrero E, et al. (1999). New electrochemiluminescent immunoassay for the determination of CYFRA21-1: analytical evaluation and clinical diagnostic performance in urine samples of patients with bladder cancer. Clin. Chem. 45: 1944-1953.

Stieber P, Dienemann H, Hasholzner U, Fabricius PG, et al. (1994). Comparison of CYFRA21-1, TPA and TPS in lung cancer, urinary bladder cancer and benign diseases. Int. J. Biol. Markers 9: 82-86.

Theyer G, Dürer A, Theyer U, Haberl I, et al. (1999). Measurements of free and total PSA, tissue polypeptide-specific antigen (TPS), and CYFRA21-1 in prostate cancer patients under intermittent androgen suppression therapy. Prostate 41: 71-77.

van Rhijn BW, van der Poel HG and van der Kwast TH (2005). Urine markers for bladder cancer surveillance: a systematic review. Eur. Urol. 47: 736-748.

von Elm E, Altman DG, Egger M, Pocock SJ, et al. (2007). STROBE Initiative: The Strengthening the Reporting of Observational Studies in Epidemiology (STROBE) statement: guidelines for reporting observational studies. Epidemiology 18: 800-804.

Washino S, Hirai M, Matsuzaki A and Kobayashi Y (2011). Clinical usefulness of CEA, CA19-9, and CYFRA21-1 as tumor markers for urothelial bladder carcinoma. Urol. Int. 87: 420-428. 
Zhang L, Liu JL, Zhang YJ and Wang H (2011). Association between HLA-B27 polymorphisms and ankylosing spondylitis in Han populations: a meta-analysis. Clin. Exp. Rheumatol. 29: 285-292.

Zintzaras E and Ioannidis JP (2005). Heterogeneity testing in meta-analysis of genome searches. Genet. Epidemiol. 28: 123-137. 